\title{
CONF-970646-2
}

\section{Dual Axis Operation of a Micromachined Rate Gyroscope}

\author{
Thor Juneau*, A. P. Pisano**, Jim Smith*** \\ *BSAC, 497 Cory Hall, Berkeley, CA 94720, thorj@nobelium.me.berkeley.edu \\ **Dept. of Mechanical Engineering, U.C. Berkeley, Berkeley, CA 94720 \\ *** Sandia National Laboratories P.O. Box 5800, Albuquerque, NM 87185
}

\section{SUMMARY}

Since micromachining technology has raised the prospect of fabricating high performance sensors without the associated high cost and large size, many researchers $[1,2,3,4]$ have investigated micromachined rate gyroscopes. The vast majority of research has focused on single input axis rate gyroscopes, but this paper presents work on a dual input axis micromachined rate gyroscope. The key to successful simultaneous dual axis operation is the quad symmetry of the circular oscillating rotor design. Untuned gyroscopes with mismatched modes yielded random walk as low as $10 \%$ hour with cross sensitivity ranging from $6 \%$ to $16 \%$. Mode frequency matching via electrostatic tuning allowed performance better than $2 \%$ hour, but at the expense of excessive cross sensitivity.

Keywords: Gyroscope, rate sensor, surface micromachining

\section{INTRODUCTION}

Conventional technology has successfully produced high performance rate gyroscopes for many years. Unfortunately, the price paid for performance is large size, high cost, and substantial power drain. Until now, the high cost of even medium performance rate gyroscopes has precluded their use in most consumer products and high volume military applications, such as automatic vehicle braking systems, augmented GPS navigation, virtual reality, and inexpensive munitions guidance. The miniature size and low power prerequisites for applications ranging from micro-satellite attitude control to battery powered personal navigators render conventional technology uncompetitive. Hence, there is clear need for inexpensive, miniature gyroscopes.

Recognizing this unfulfilled need, many researchers have pursued the goal of devising a micromachined rate gyroscope. The inherent size, weight, power, and cost advantages of MEMS should allow micromachined rate gyroscopes to fill the void left by conventional technology. Solid state sensor reliability and robustness to shock and to vibration makes micromachining even more attractive.

The majority of published micro-gyroscope designs are single input axis sensors based on either translational vibration $[1,2]$ or structural mode vibration[3]. Few of these designs are compatible with integrated VLSI processing. In contrast, this research focused on fabricating a dual input axis gyroscope using the fully integrated Analog Devices Inc. surface micromachining process [5]. The on chip interface and signal processing allows improved noise performance, extreme miniaturization, and inexpensive manufacture. In addition, integrated surface micromachining enables multiple sensors on the same substrate. Combining this dual axis gyroscope with a $\mathrm{z}$-axis gyroscope $[1,3]$ yields angular rate measurement in all orthogonal axes. By also adding a triad of micro-accelerometers, a thumb-nail sized Inertial Measurement Unit (IMU) has been designed and is being fabricated at Sandia National Laboratories [8].

\section{MECHANICAL STRUCTURE}

The basic operating principle is based on the generation and detection of a Coriolis angular acceleration. A $2 \mu \mathrm{m}$ thick polysilicon disk with a $150 \mu \mathrm{m}$ radius serves as an inertial rotor. As depicted in Fig. 1, this inertial rotor is suspended $1.6 \mu \mathrm{m}$ above the substrate by four symmetrically placed beams anchored to the substrate. These beams provide a torsional suspension allowing rotational compliance about all three axes.

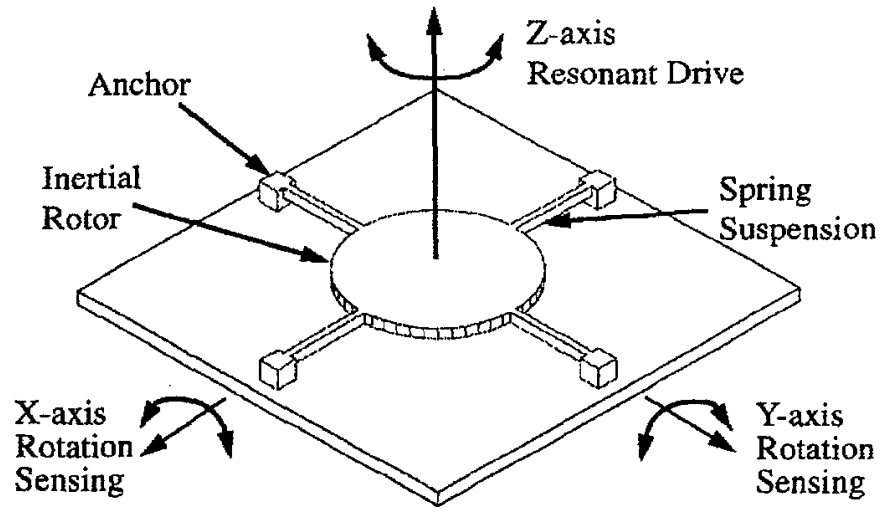

Fig. 1: Conceptual illustration of dual axis gyroscope.

In order to generate a Coriolis acceleration, the inertial rotor is driven into angular resonance about the $z$-axis perpendicular to the substrate. When the inertial rotor is resonating, any rotation rate of the substrate about the $x$-axes will induce a Coriolis angular acceleration about the $y$-axis which in turn induces a tilting oscillation of the rotor about the $y$-axis. Because the mechanical gyroscope is symmetrical in two orthogonal axes, the sensor is also responsive to rotation rate about the $y$-axis. A rotation rate input about the $y$-axis invokes a tilting oscillation output about the $x$-axis thereby allowing dual axis rotation rate measurement. These dynamics are demonstrated in the equations for Coriolis acceleration $\alpha$ on each axis where $I$ is the respective moment of inertial, $\omega_{z}$ is the resonant drive frequency, $X_{0}$ is the resonant drive amplitude, $\Omega$ is an input rotation rate to be measured, and $t$ is time.

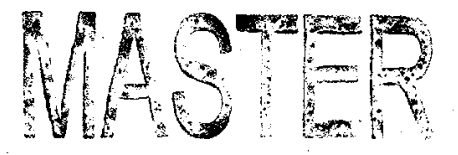




\section{DISCLAIMER}

This report was prepared as an account of work sponsored by an agency of the United States Government. Neither the United States Government nor any agency thereof, nor any of their employees, make any warranty, express or implied, or assumes any legal liability or responsibility for the accuracy, completeness, or usefulness of any information, apparatus, product, or process disclosed, or represents that its use would not infringe privately owned rights. Reference herein to any specific commercial product, process, or service by trade name, trademark, manufacturer, or otherwise does not necessarily constitute or imply its endorsement, recommendation, or favoring by the United States Government or any agency thereof. The views and opinions of authors expressed herein do not necessarily state or reflect those of the United States Government or any agency thereof. 


\section{DISCLAMERR}

Portions of this document may be illegible in electronic image products. Images are produced from the best available original document. 


$$
\begin{array}{ll}
X \text {-axis } & \alpha_{\text {Corr }}^{X}=2 \cdot I_{X} \cdot \Omega_{Y} \cdot \omega_{Z} \cdot \operatorname{Cos}\left(\omega_{Z} t\right) \\
\text { Y-axis } & \alpha_{\text {Corr }}^{Y}=2 \cdot I_{Y} \cdot \Omega_{X} \cdot \omega_{Z} \cdot \operatorname{Cos}\left(\omega_{Z} t\right)
\end{array}
$$

The tilting oscillations about the $x$ - and $y$-axis are at the same frequency as the resonant drive. These tilting oscillations are amplitude modulated signals with amplitude proportional to the respective rotation rate inputs. Therefore, the rotation rate can be inferred by capacitively detecting the rotor tilting oscillation and demodulating with the resonant drive signal.

A micrograph of the dual axis gyroscope is shown in Fig 2. At the center is the circular inertial rotor suspended by four suspension beams extending radially from the rotor's outside perimeter. The beams are $180 \mu \mathrm{m}$ in length with some stress relief at the outside edges. An external suspension, as opposed to a spoked wheel internal suspension inside the rotor, was chosen to accommodate warping due to large residual stress gradients. To maximize mechanical sensitivity, the suspension was designed to match the frequencies of all three rotational modes sufficiently well so that electrostatic tuning may compensation for fabrication process variation. Specifics regarding suspension design trade offs revolving around sensitivity, shock survival, and electrostatic forces can be found in references $[6,7]$.

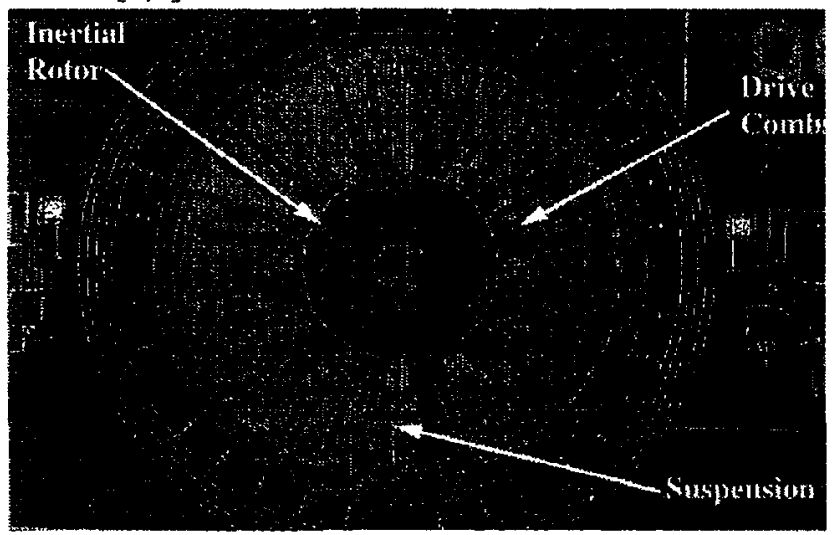

Fig. 2: Close-up die photo showing open-loop micromachined gyro design with signal bus and drive electronics.

There can be no rotation rate sensing unless the inertial rotor is driven into rotational resonance. This task is accomplished using highly linear electrostatic comb drive [7]. The twelve pairs of alternating differential drive and sense combs can be seen surrounding the rotor in Fig 2 above. A differential trans-resistance amplifier provides positive feedback to the comb drive effectively cancelling viscous damping and inducing rotational resonance. Since the amplitude of resonance directly determines the scale factor, an automatic gain control loop is used to ensure constant oscillation amplitude [6]. The present circuitry requires the gyroscope be operated in ambient pressure below $150 \mathrm{~m}$ Torr. A trans-resistance gain of approximately $3 \mathrm{M} \Omega$ is required for oscillation at $60 \mathrm{mTorr}$.

\section{SINGLE AXIS OPERATION}

With the basic mechanical structure and operating princi- ple described, attention is now focused on single axis operation. As related previously, a rotation rate input about the $\mathrm{x}$-axis induces a Coriolis acceleration which in turn induces a tilting oscillation about the $\mathrm{y}$-axis. By capacitively measuring this tilt oscillation amplitude, the original rotation rate input can be inferred. The differential capacitive measurement system is illustrated in Fig 3 for a single axis. The side view looking beneath the rotor reveals a pair of quarter-pie shaped $\mathbf{n}+$ diffusion electrodes which form a capacitive divider with the inertial rotor. If the rotor tilts about the axis perpendicular to the page, then the capacitance of one sense capacitor increases while the capacitance of the other decreases. This differential change in capacitance is detected via an integrator $\left(\mathrm{C}_{\mathrm{I}}=50 \mathrm{fF}\right)$ attached to the inertial rotor in conjunction with a modulated sense voltage applied between the pair of quarter-pie shaped electrodes.

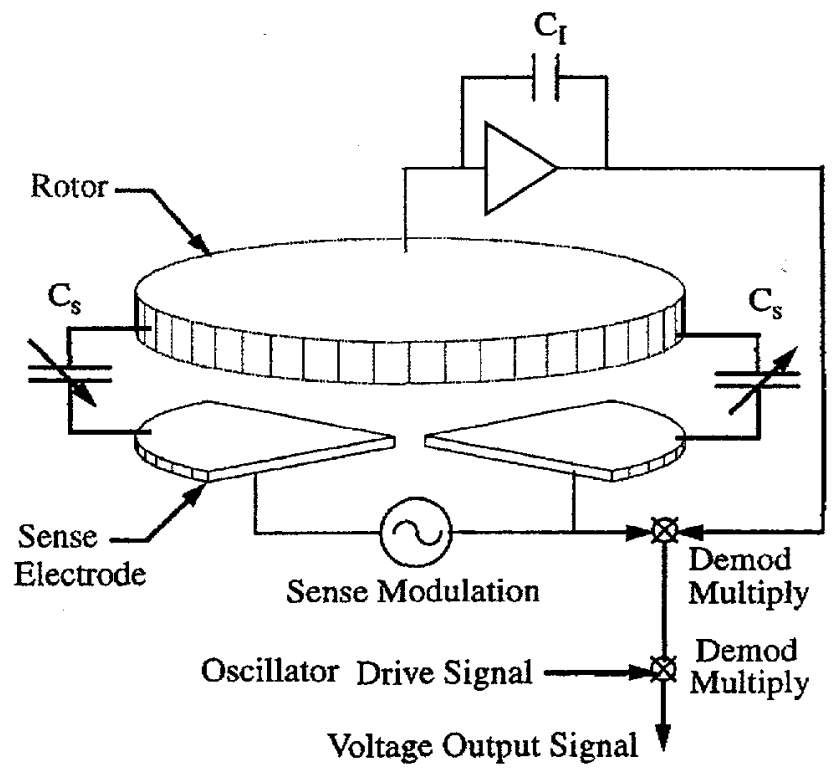

Fig. 3: Side view showing rotor with underlying diffusion sense electrodes for one sense axis.

The voltage output from the integrator must be demodulated twice to recover the desired voltage output signal [6]. First, a demodulation removes the sense voltage modulation frequency leaving a voltage proportional to inertial rotor tilt position. Second, a demodulation removes the inertial rotor drive frequency leaving a base band voltage signal proportional to rotation rate input. This voltage signal is the desired output, hence explanation of single axis operation is concluded. Now dual axis operation is considered.

\section{DUAL AXIS OPERATION}

As pointed out previously, the dual axis rate gyroscope is $i$ equally sensitive to rotation rates about both the $x$ - and $y$-axis. Differentiating between $x$ - and $y$-axis input rotation rates is accomplish by differentiating between the orthogonal $x$ - and $y$ axis inertial rotor tilt oscillations due to Coriolis acceleration. To this end, two pairs of quarter-pie shaped electrodes are patterned beneath the inertial rotor. As illustrated in Fig. 4, each 
orthogonal pair of diametrically opposed quarter-pie shaped electrodes allows detection of tilt oscillation about the orthogonal $x$-and $y$-axis.

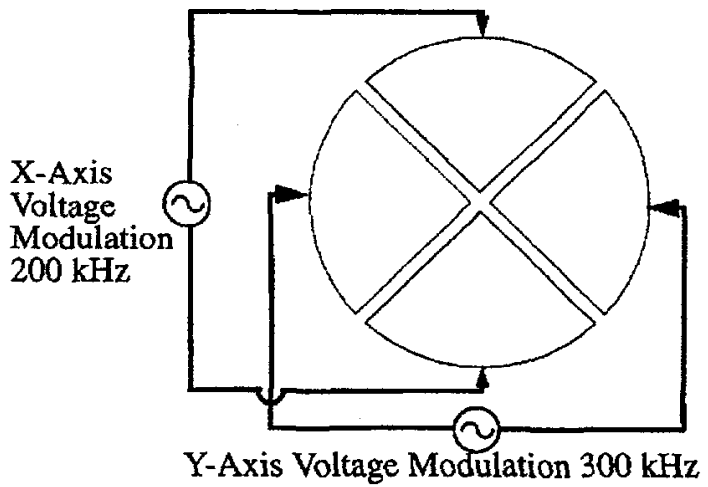

Fig. 4: Top view showing four diffusion sense electrodes beneath inertial rotor. Note $x$ - and $y$-axis have different modulation frequencies so two rotation rates may be independently resolved.

Because all tilt oscillation detection is completed using a single integrator attached to the structure, electrical differentiation between $\mathrm{x}$ - and $\mathrm{y}$-axis tilt oscillation is accomplished by using a different sense modulation frequency for each axis. Separate demodulation circuits for each axis provide two output signals proportional to the two orthogonal rotation rate inputs.

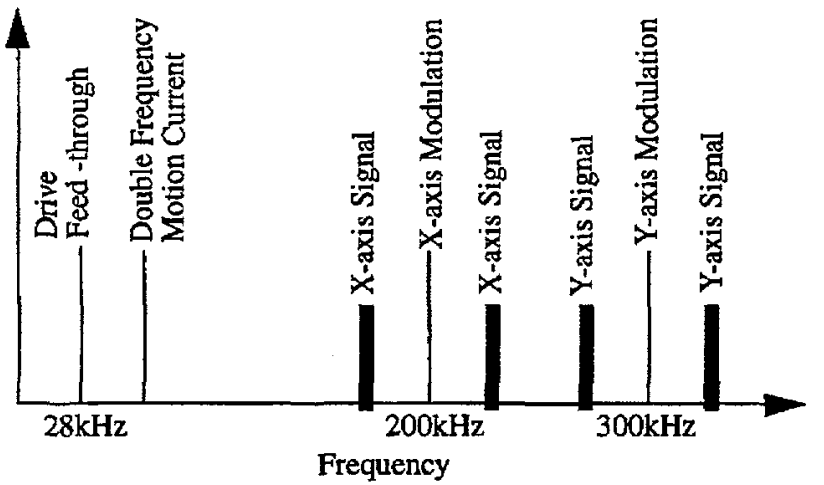

Fig. 5: Conceptual Power Spectrum plot showing gyroscope signal distribution over frequency.

As the multiple signal frequency bands shown in Fig. 5 suggest, choosing sense modulation frequencies demands great care. Clearly, the sense modulation voltages should have a far higher frequency than the inertial rotor resonance to avoid mixing with drive feed-though, double frequency motion current, and any drive signal distortion. Since the integrator output signals for each axis are actually double modulated, the detection signals for each axis appear as double side bands spaced equal distant about each respective modulation voltage frequency. The frequency difference between each side band and the original sense modulation voltage is equal to the drive frequency ( $28 \mathrm{kHz}$ in this case) because the Coriolis acceleration induced tilt oscillation is at the drive frequency. These side bands should never mix, so the sense voltage modulation frequencies have a minimum separation of greater than twice the drive resonant frequency. In addition, higher harmonics resulting from distortion can interfere with signal purity, so frequencies of $200 \mathrm{kHz}$ and $300 \mathrm{kHz}$ were chosen.

\section{EXPERIMENTAL RESULTS}

The rotation rate sensing performance of several dual axis gyroscope devices was tested using a miniature vacuum chamber mount atop a Contraves rate table. In addition to performance, those parameters with greatest impact on performance such as natural frequency and quality factor $Q$, have been identified as summarized by Table 1 . Vacuum chamber operation results in a high $Q$ resonant peak which allows significant improvements in sensitivity via matching drive resonant frequency with sense mode natural frequency. Due to process yariation, a distribution of poorly-matched and nearly matched sensors could be compared. The dominant random walk source was electrical interface noise, so the devices with poorly. matched modes and lower sensitivity exhibited $110 \%$ h hour sandom walk, while the nearly matched mode devices with better sensitivity exhibited $10 \%$ hour random walk. A key parameter in all sensors, but especially dual axis sensors is cross sensitivity. During open-loop operation the devices with better mode matching and hence better noise performance showed larger cross sensitivity. This is not unexpected as operating open-loop with mode matching can exasperate cross coupling as revealed in the next section.

Table 1: Experimental Results

\begin{tabular}{|l|l|l|}
\hline \multicolumn{1}{|c|}{ Parameter } & \multicolumn{1}{|c|}{$\begin{array}{c}\text { Poorly- } \\
\text { Matched }\end{array}$} & \multicolumn{1}{|c|}{$\begin{array}{c}\text { Nearly- } \\
\text { Matched }\end{array}$} \\
\hline \hline Drive Frequency & $28.2 \mathrm{kHz}$ & $28.4 \mathrm{kHz}$ \\
\hline Frequency Matching Error & $8.8 \%$ & $1.4 \%$ \\
\hline Quality Factor $\mathrm{Q}$ & 960 & 950 \\
\hline Scale Factor $(\mu \mathrm{V}$ per $\% \mathrm{sec})$ & 190 & 2200 \\
\hline Cross Sensitivity & $6 \%$ & $16 \%$ \\
\hline
\end{tabular}

\section{CROSS-AXIS SENSITIVITY}

Matching drive and sense modes vastly enhances noise performance, but cross sensitivity may consequently increase during open-loop operation. Part of this may be due to slight differences in $\mathrm{x}$ - and $\mathrm{y}$-axis natural frequencies. However, mechanical cross coupling is the most important culprit. Asymmetries and irregularities stemming from fabrication imperfections can result in inertial, viscous, and elastic cross coupling. Since the dual axis gyroscope beams are minimum process dimensions, elastic cross coupling is the most likely candidate. Elastic cross coupling simply implies that the suspension restoration torque is a function of both $\mathrm{x}$ - and $\mathrm{y}$-axis angular displacements $\theta_{\mathrm{x}}$ and $\theta_{\mathrm{y}}$ as denoted in Eq. 3 and Eq. 4.

$$
\begin{aligned}
& T_{E \text { Elastic }}^{X}=K \theta_{X}+\delta \times K \theta_{Y} \\
& T_{\text {Elastic }}^{Y}=K \theta_{Y}+\delta \times K \theta_{X}
\end{aligned}
$$


Assuming for simplicity that the sense axes are identical, $\mathrm{K}$ is the standard spring constant and $\delta \mathrm{K}$ is the elastic cross coupling term equal to the standard spring constant multiplied by a coupling coefficient $\delta$. Adding the dynamical effects of mode matching, the following approximate function for cross sensitivity $S_{C r o s s}$ is derived where $Q$ is the quality factor, $\omega_{\text {sense }}$ is the sense mode frequency, and $\omega_{\text {drive }}$ is the drive resonant frequency.

$$
S_{\text {Cross }}=\frac{\delta \omega_{\text {Sense }}^{2}}{\omega_{\text {Drive }}^{2}+\left(\omega_{\text {Drive }} \omega_{\text {Sense }}\right) / Q+\omega_{\text {Sense }}^{2}}
$$

This function is the exact same shape as a typical second order system with low damping and a resonance peak. When mode matching is exact, cross sensitivity due to cross coupling is maximized. This was experimental observed when electrostatic frequency tuning was used to precisely match modes. This resulted in noise performance of $2 \%$ hour, but with excessive cross sensitivity. This degradation of cross sensitivity is a consequence of open-loop dynamics which can be suppressed by closed-loop force balancing as discussed next.

\section{SOLUTION: CLOSED-LOOP FORCE BAL- ANCING CONTROL}

Under open-loop operation, matching natural frequencies closely has several dynamical drawbacks. There is an underlying trade off between noise performance and cross sensitivity as afore mentioned. Because gain and phase change radically at the resonant peak, scale factor is not constant over bandwidth and frequency drift induces both scale factor and output phase changes. These consequences limit practical mode matching for open-loop operation to below $5 \%$.

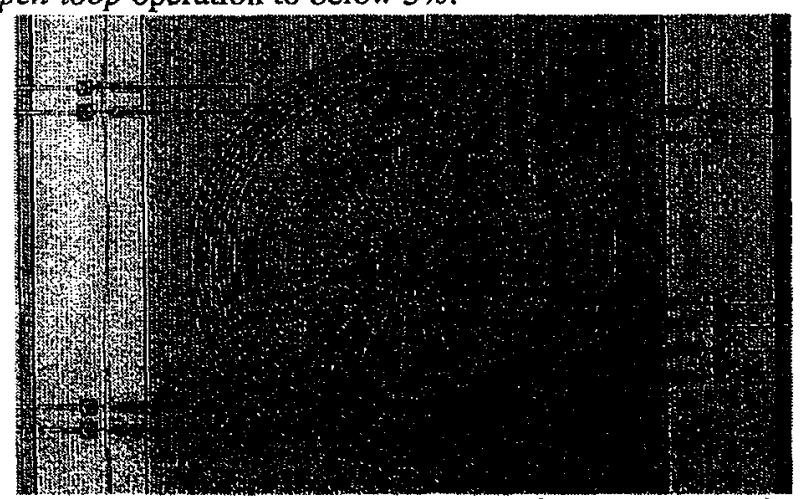

Fig. 6: Next generation dual axis gyro design fabricated at Sandia National Labs features force balancing capability.

However, closed-loop feedback is often used to enhance performance beyond the limitations of open-loop operation. Feedback typically improves scale factor stability, linearity, bandwidth, and operating range. A well designed feedback loop will not significantly alter noise performance. Thus the noise advantages of mode matching can be retained, while the dynamical advantages of closed-loop feedback can be added. In fact, closed-loop force balancing of the dual axis gyroscope will alle- viate most scale factor and phase difficulties while also reducing cross axis sensitivity significantly below the $6 \%$ attained thus far. Improved offset stability will be achieved by cancelling quadrature error (rotor wobble due to imbalance) using a feedback loop driving special comb electrodes designed for levitation forcing. The added electrodes for both these feedback loops have been incorporated into the next generation dual axis gyroscope design (see Fig. 6) which is fabricated by Sandia National Labs [8].

\section{CONCLUSION}

A dual axis rate gyroscope which measures rotation rate inputs about two input axes simultaneously has been design, fabricated, and tested. Good open-loop performance was achieved, but the peak performance via mode matching resulted in degradation of cross sensitivity. However, closed-loop force balancing should allow future designs to benefit from the performance advantages of mode matching without sacrificing cross sensitivity and scale factor stability.

\section{ACKNOWLEDGEMENTS}

This work was funded by DARPA grant TP-26890. No progress would have been possible without fabrication completed at Analog Devices Inc. and Sandia National Labs. Sandia is a multi-program laboratory operated by Sandia Corporation, a Lockheed Martin Company, for the United States Department of Energy under contract DE-AC04-94AL85000. The first author would like to thank Bill Clark for his kind circuitry help.

\section{REFERENCES}

[1] W. A. Clark, R. T. Howe, R. Horowitz, "Surface Micromachined Z-axis Vibratory Rate Gyroscope", Hilton Head '96, pp. 283.

[2] Paul Greiff et al, "Vibrating Wheel Micromechanical Gyro", IEEE "96 Position location \& Navigation Symposium, pp. 31 .

[3] M. Putty \& K. Najafi, "A Micromachined Vibratory Ring Gyroscope", Hilton Head '94, pp. 213.

[4] Tony K. Tang, "A Packaged Silicon MEMS Vibratory Gyroscope for Microspacecraft", MEMS '97, Japan, pp. 500.

[5] R. S. Payne, S. Sherman, S. Lewis, R. T. Howe, "Surface Micromachining: From Vision to Reality to Vision", 1995 IEEE International Solid State Circuits Conference, pp. 164.

[6] T. Juneau, A. P. Pisano, "Micromachined Dual Input Axis Angular Rate Sensor", Hilton Head '96, pp. 299.

[7] P. B. Ljung, T. Juneau, A. P. Pisano, "Micromachined Two Input Axis Angular Rate Sensor", ASME International Mechanical Engineering Congress and Exposition 1995, session DSC-16.

[8] Jim Smith et al, "Embedded Micromechanical Devices for the Monolithic Integration of MEMS with CMOS", Proc. 1995 IEDM, pp, 609-612. 